\title{
CRISPR-Cas-mediated chromosome engineering for crop improvement and synthetic biology
}

\author{
Michelle Rönspies ，Annika Dorn, Patrick Schindele and Holger Puchta 凶
}

\begin{abstract}
Plant breeding relies on the presence of genetic variation, as well as on the ability to break or stabilize genetic linkages between traits. The development of the genome-editing tool clustered regularly interspaced short palindromic repeats (CRISPR)CRISPR-associated protein (Cas) has allowed breeders to induce genetic variability in a controlled and site-specific manner, and to improve traits with high efficiency. However, the presence of genetic linkages is a major obstacle to the transfer of desirable traits from wild species to their cultivated relatives. One way to address this issue is to create mutants with deficiencies in the meiotic recombination machinery, thereby enhancing global crossover frequencies between homologous parental chromosomes. Although this seemed to be a promising approach at first, thus far, no crossover frequencies could be enhanced in recombination-cold regions of the genome. Additionally, this approach can lead to unintended genomic instabilities due to DNA repair defects. Therefore, efforts have been undertaken to obtain predefined crossovers between homologues by inducing site-specific double-strand breaks (DSBs) in meiotic, as well as in somatic plant cells using CRISPR-Cas tools. However, this strategy has not been able to produce a substantial number of heritable homologous recombination-based crossovers. Most recently, heritable chromosomal rearrangements, such as inversions and translocations, have been obtained in a controlled way using CRISPR-Cas in plants. This approach unlocks a completely new way of manipulating genetic linkages, one in which the DSBs are induced in somatic cells, enabling the formation of chromosomal rearrangements in the megabase range, by DSB repair via non-homologous end-joining. This technology might also enable the restructuring of genomes more globally, resulting in not only the obtainment of synthetic plant chromosome, but also of novel plant species.
\end{abstract}

A $\mathrm{s}$ the growing world population faces limited food resources-for example, due to restricted availability of farmland or instability of yields - the development of novel and fast ways to improve current crops is essential for meeting future food demands. Examples of such improvements are increased yields, nutritional value or tolerance to biotic and abiotic stresses ${ }^{1}$. This process can take several years with conventional breeding techniques and is limited by factors such as linkage drag-that is, linked inheritance of undesired genetic material associated with favourable traits-or reduced genetic diversity due to the domestication process $^{2}$. Examples of linkage drag in crop plants are seen in the association of resistance to tobacco mosaic virus with reduced yield in tobacco and the association of resistance to Fusarium wilt with increased sensitivity to bacterial spot in tomato ${ }^{3,4}$. The development of clustered regularly interspaced short palindromic repeats (CRISPR)-CRISPR-associated protein (Cas) technology as a genome-editing tool has led to a new era in crop improvement ${ }^{5-9}$. The CRISPR-Cas system consists of a DNA endonuclease that is able to induce double-strand breaks (DSBs) at almost any location in the genome and is guided to the desired cut site by a synthetic single guide $\mathrm{RNA}^{10}$ (sgRNA). The induced DSBs are then repaired by endogenous DNA repair pathways, such as non-homologous end-joining (NHEJ) or homology-directed repair (HDR) ${ }^{11}$. The NHEJ pathway, which is predominant in somatic cells, is error-prone and can lead to small insertions or deletions at the break site ${ }^{11}$. If two or more DSBs are induced simultaneously, NHEJ repair can also lead to chromosomal rearrangements such as deletions, inversions or translocations ${ }^{12,13}$. In mammals, natural chromosomal rearrangements are mainly associated with the development of cancer and are the cause of several genetic disorders ${ }^{14-16}$. Chromosomal rearrangements that mutate a tumour suppressor gene or activate an oncogene can lead to cancer ${ }^{17}$. Disorders caused by chromosomal rearrangements include Hunter syndrome, Emery-Dreifuss muscular dystrophy and haemophilia $\mathrm{A}^{18-21}$. Conversely, chromosomal rearrangements have an important role in adaptation and speciation $^{22}$, especially in plants. One reason for this is that heterozygous chromosomal rearrangements can lead to the generation of unbalanced gametes during meiotic recombination and can affect fertility or cause reproductive isolation ${ }^{22,23}$. Chromosomal rearrangements also have diverse effects on the regulation of gene expression, which can affect the whole genome $\mathrm{e}^{24}$. In heterozygotes, genetic information contained in chromosomal inversions and translocations is not available for gene reshuffling, as meiotic recombination is supressed within the rearranged area ${ }^{25,26}$. Chromosomal rearrangements can thus complicate the breeding process when genetic exchange between two cultivars is suppressed. To date, only small-scale genomic changes have been induced in plants using CRISPR-Cas. However, the induction of large, megabase-scale chromosomal rearrangements has been reported in several recent studies in both Arabidopsis thaliana and maize ${ }^{27-29}$. In this Review, we highlight chromosome engineering as an attractive novel tool for breaking or stabilizing genetic linkages in the field of plant breeding. Furthermore, on the basis of recent research, we discuss the prospects of alternative approaches to inducing recombination between homologous chromosomes.

Manipulating DNA repair pathways to break genetic linkages in plant breeding

Plant breeding relies on crossovers, the reciprocal exchange of genetic material, between homologous chromosomes during meiosis to generate new allelic combinations. Crossovers allow favourable traits to be combined and adverse traits to be eliminated from 
elite crops. However, since crossover rate and distribution in the natural process are highly restricted and not very controllable, major parts of the chromosomes do not participate in the genetic exchange $^{30}$. Consequently, desirable recombination outcomes are highly limited and linkage drag is frequently inevitable. During meiosis, parental genome sets are halved during two consecutive nuclear divisions, without interstitial replication to generate haploid gametes ${ }^{31}$. The physical connection of the paired homologous chromosomes, termed bivalents, via crossovers is essential for the correct segregation of the homologous chromosomes during the first meiotic division. In all organisms, this is ensured by the maintenance of one obligatory crossover per chromosome pair, which is the minimum crossover number for recombination processes. Although meiotic recombination is the basis of genetic variance in the offspring, crossover numbers remain within a tight range in plants, rarely exceeding three crossovers per bivalent ${ }^{32}$.

The starting point for meiotic recombination is the programmed induction of DSBs by highly conserved SPO11 homologues ${ }^{33}$. The specifics of the subsequent meiotic DSB repair mechanisms define the occurrence of crossovers ${ }^{31}$ (Fig. 1). For the formation of the displacement loop (D-loop), which is the first repair intermediate leading to crossover formation, DSB ends are resected and strand invasion into homologous sequences (preferably the homologous chromosome) occurs. Synthesis-dependent strand annealing (SDSA) is an important mechanism for the formation of non-crossovers (NCOs), whereby the invaded strand is elongated. It is then rejected from the D-loop and spans the DSB for repair synthesis. Conversely, the D-loop can also be extended, so that the discarded strand can anneal with the opposite side of the DSB in a second-end capture. This enables the formation of the second key intermediate, the double Holliday junction $(\mathrm{dHJ})$. The resolution of this structure is the only mechanism in crossover formation in which resolvases dissolve the $\mathrm{dHJ}$ by endonucleolytic cleavage. Multiple factors have been identified in plants that shift the equilibrium of crossover genesis in one direction or the other. Exploiting this knowledge by mutating crossover-limiting or by overexpressing crossover-promoting factors is a promising method for increasing genetic variance in plant breeding.

The first crossover-limiting factor discovered in plants was the helicase Fanconi anaemia complementation group M (FANCM). Mutations in fancm resulted in a threefold increase of crossover frequency in $A$. thalian $a^{34}$. The second helicase, identified as a crossover antagonist, is the BLM homologue RECQ4. Arabidopsis possesses two closely related homologues, RECQ4A and RECQ4B ${ }^{35}$. The recq $4 a$ recq $4 b$ (hereafter recq $4 a / b$ ) double mutant has shown a 6.2-fold increase in crossover frequency ${ }^{36}$. Both RECQ4 and FANCM have roles in the destablization of D-loops to promote SDSA. Thus, the simultaneous mutation of these helicases was postulated to reflect an almost complete absence of SDSA. This would indicate that all DSBs result in $\mathrm{dHJ}$-like structures that are resolved by resolvases, equally resulting in crossovers and NCOs. Furthermore, RECQ4 homologues probably act in the evolutionary conserved RecQ-Top3-Rmi (RTR) complex, which is involved in the dissolution of Holliday junction-like intermediates ${ }^{37}$, as similar crossover-limiting functions have been found in the other complex partners, Topoisomerase $3 \alpha$ (TOP $3 \alpha$ ) and RecQ-mediated genome instability 1 (RMI1), in Arabidopsis ${ }^{38}$. Indeed, CRISPRCas9-mediated mutagenesis of RECQ4 in a Solanum lycopersicum and Solanum pimpinellifolium hybrid resulted in a 1.8-fold increase in crossovers $^{39}$.

Additionally, other factors contribute to crossover control in plants. The conserved AAA-ATPase FIDGETIN-LIKE-1 (FIGL1), together with its complex partner Fidgetin-like-1 interacting protein (FLIP), is involved in the control of recombinases for the initiation of strand invasion ${ }^{40,41}$. Mutations in Arabidopsis figll have been shown to increase crossover numbers in both inbred and hybrid

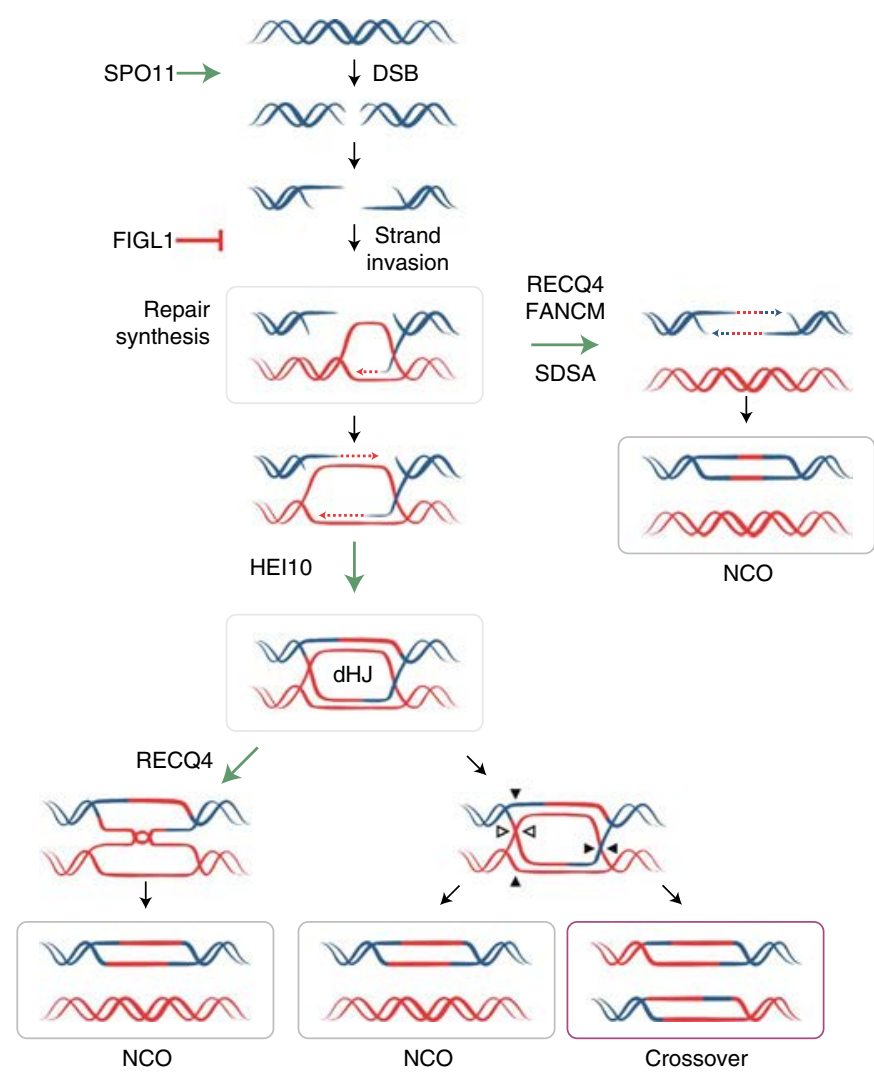

Fig. 1 | Enhancement of meiotic crossovers by modulation of crossover control factors. Meiotic recombination is induced by DSBs followed by resection of the break ends. FIGL1 is a negative regulator of strand invasion, whereby single-stranded overhangs invade into homologous sequences. Repair synthesis elongates the invaded strand, resulting in the formation of a D-loop. In the SDSA pathway, the helicases RECQ4 and FANCM promote the D-loop disassembly to generate an NCO outcome. The stabilization and extension of the D-loop results in a second-end capture, whereby the discarded strand anneals with the opposite side of the DSB. Repair synthesis and ligation lead to the formation of a $\mathrm{dHJ}$ structure. The E3 ligase $\mathrm{HEI} 10$ is involved in the stabilization of recombination intermediates, the precursors of $\mathrm{dHJs}$. The $\mathrm{dHJ}$ can be processed by RECQ4, generating a hemicatenane-intermediate that can be further dissolved to form an NCO product. By contrast, the nucleolytic cleavage of the $\mathrm{dHJ}$ by resolvases results in the formation of both crossover and NCO products.

lines. The downside of using figl1 mutants in crops is their sterility, which has been observed in rice, pea and tomato mutants, resulting in a breeding dead end ${ }^{42,43}$. The alternative to using mutants of anti-crossover factors to achieve an increase in crossover frequency is the overexpression of crossover-promoting factors, such as the E3 ligase Human enhancer of invasion 10 (HEI10) ${ }^{44}$. In Arabidopsis, the artificial overexpression of HEI10 leads to an increase in crossover frequencies, especially in subtelomeric euchromatin ${ }^{45}$. However, when combined with a recq $4 a / b$ mutant background, the observed crossover increase was again restricted to the chromosome arms, with the highest effect in subtelomeres, but with no effect on centromeres ${ }^{46}$. Interestingly, the mutation of ZEP1, a synaptonemal complex protein in rice, resulted in an increase of crossovers, similar to HEI10 overexpression, indicating that further factors could be identified $^{47}$. Since multiple pathways influence crossover abundance, the combination of different strategies promises a further boost of crossover frequency. For example, the simultaneous mutation of figl 1 and recq $4 a / b$, or fancm and recq $4 a / b$, resulted in a tenfold increase in crossovers in Arabidopsis inbred strains. A 7.8-fold increase was 


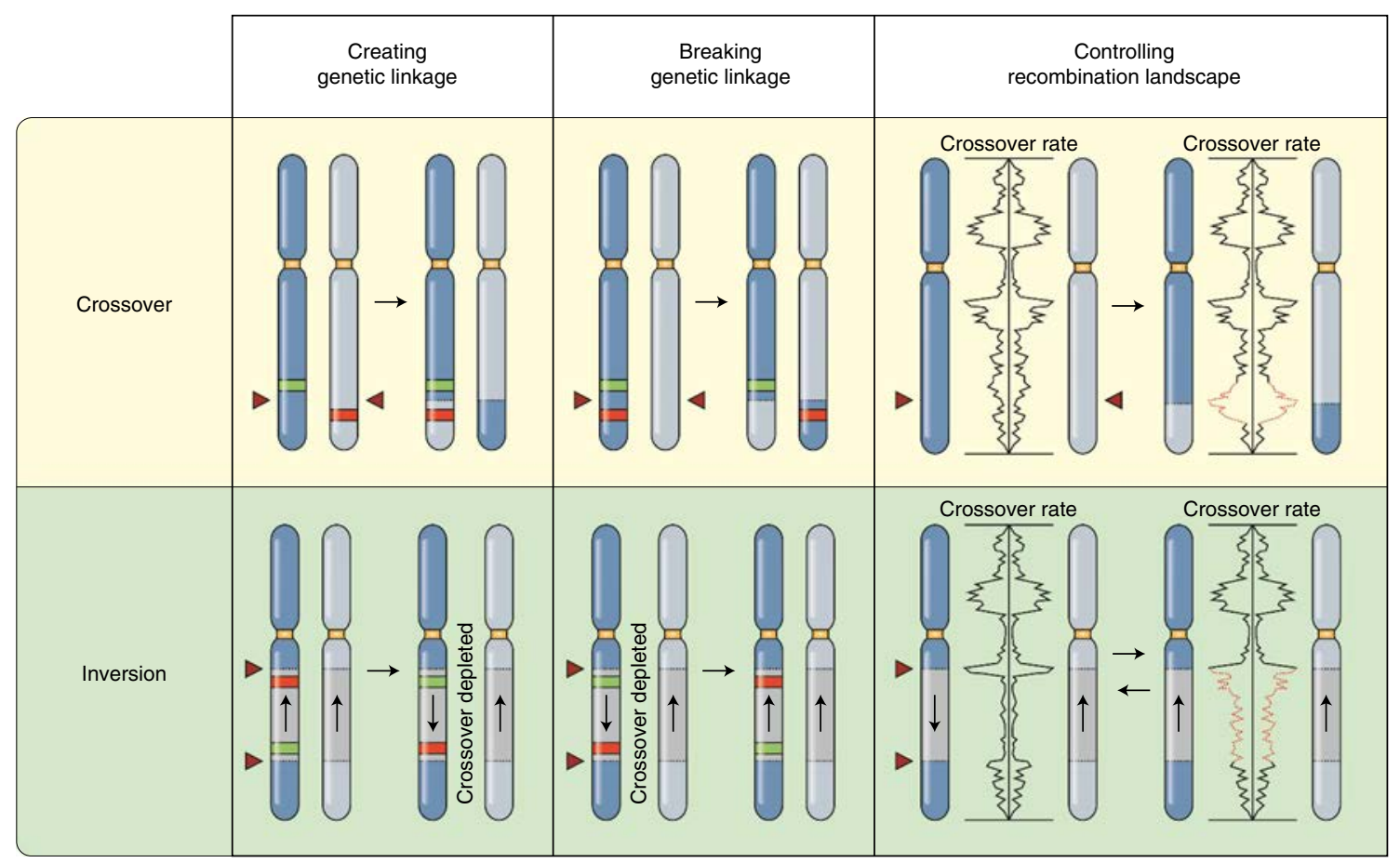

Fig. 2 | Control of genetic exchange by targeted induction of crossovers and inversions. Linkage drag is caused by a lack of genetic exchange in between the respective traits. Consequently, traits in close proximity or traits that reside within an inverted region on the same chromosome are, with high probability, genetically linked. The targeted induction of crossovers and inversions enables control of these types of linkages. Artificial crossovers between homologous chromosomes could break the linkage of traits (green and red boxes) linked by close proximity (top centre). Alternatively, artificial crossovers could be used to establish new linkage groups by joining traits on the same chromosome in close proximity (top left). Naturally inverted and thus crossover-depleted regions could be reversed to re-establish genetic exchange and consequently unlock formerly linked traits (bottom centre). Additionally, artificially induced inversions could prevent regions from genetic exchange by promoting linkage groups (bottom left). Both chromosomal rearrangements pave the way to controlling and manipulating the natural recombination landscape in a targeted manner (top right and bottom right). Red arrowheads indicate CRISPR-Cas-mediated DSBs.

detected in recq4a/bfigl 1 triple mutants. As no further rise was observed in a quadruple mutant with fancm, it was postulated that some sort of threshold might have been reached ${ }^{48}$. The possibility of transferring these findings to various crop plants was demonstrated in a study from 2018 that identified RECQ4 homologues as a central determinant of crossover frequency. Inactivation of RECQ4 homologues in rice, pea and tomato hybrids resulted in increases of 3.2-, 4.7- and 2.7-fold in crossover frequency, respectively, showing the potential of accelerating breeding processes using mutants ${ }^{43}$. Although the possibilities of manipulating crossover pathways to increase meiotic recombination seem very auspicious, there must be a note of caution when manipulating DNA repair factors. The most promising strategy for mutating RECQ4 homologues comes at a price. RECQ4, which is part of the RTR complex, is not only an important factor in crossover control, but also fulfils essential functions in the maintenance of somatic genome stability ${ }^{35,49}$. For example, RECQ4A is a major factor in crosslink repair in Arabidopsis $^{50,51}$. Thus, using recq4 mutants for breeding purposes might result in an accumulation of secondary mutations and a general decrease in fitness. The same holds true for FANCM, which also has an important role in preserving genome stability in plants ${ }^{52}$. Finally, the discussed approaches for boosting crossovers were mostly only able to enhance crossover frequency. They did not have an effect on crossover positioning, leaving recombination-depleted areas, such as the pericentromeric regions, unchanged.

\section{CRISPR-Cas-mediated crossover induction}

As discussed above, the use of meiotic mutants mainly enhances crossover rates, but it does not change crossover distribution.
Therefore, a substantial part of the genome cannot be activated for recombination using this approach ${ }^{30}$. Thus, an obvious strategy is to target these regions directly to introduce DSBs during meiosis and induce homologous recombination ${ }^{53}$. In principle, two different approaches can be used to achieve this goal: using a programmable DNA nuclease for DSB induction or using its DNA-binding capability to guide the natural DSB-inducing machinery to the respective target site.

In an extensive yeast study, the adaptability of genome-editing tools to manipulate meiotic recombination was first demonstrated by using zinc finger nucleases, transcription activator-like effector nucleases and the CRISPR-Cas system as DNA-binding proteins to recruit SPO11 as a natural mediator for meiotic DSB induction $^{54}$. Guiding these SPO11 fusions to sites that naturally show low levels of DSB induction during meiosis, resulted in detectable SPO11-mediated DSB formation and a significant increase in crossover frequency. Nevertheless, regions where DSB formation is suppressed during meiosis, such as centromeric and pericentromeric regions, were still inaccessible for SPO11-mediated DSB induction, hinting at natural limitations of this system. The same approach was recently used in plants ${ }^{55}$. The SPO11 complex partner meiotic topoisomerase VIB (MTOPVIB), which is crucial for SPO11-mediated DSB induction during meiosis, was fused to dead Cas9 (dCas9) and guided to a crossover hotspot in A. thaliana that had previously been shown to be accessible for crossover manipulation. In yeast, DSB formation had been achieved by guiding SPO11 to hotspots. Therefore, it was anticipated that the crossover rate would increase with high probability. However, neither crossover frequency nor crossover distribution were significantly affected 
by dCas9-MTOPVIB. These results indicate that the recruitment of the natural DSB-inducing machinery might not be sufficient to manipulate crossover occurrence in plants.

To date, no report on the direct induction of meiotic site-specific DSBs has been published, but targeted DSB induction using Cas9 has successfully induced recombination between homologous chromosomes in somatic cells in tomato ${ }^{56}$. By making use of two genetically distinct tomato accessions, an allele-specific DSB was induced in the PHYTOENE SYNTHASE 1 gene in hybrid plants. The implementation of a fruit-colour assay enabled the differentiation between NHEJ and homologous recombination repair outcomes. Further analysis of SNP redistribution revealed that, in addition to NHEJ-mediated repair, somatic homologous recombination events also occurred. These included gene conversions and one putative crossover. Although germline transmission could only be confirmed in the case of the gene conversions, the study clearly highlighted the potential of targeted DSB induction by CRISPR-Cas in manipulating recombination between homologous chromosomes in plants.

Recently, the same group attempted a similar approach, using hybrids of genetically distinct tomato accessions - which harboured heterozygous mutations in the CAROTENOID ISOMERASE geneas a selection system to detect homologous recombination-mediated somatic recombination ${ }^{57}$. In this study, one crossover event with transitions of at least $1 \mathrm{Mbp}$ in both directions from the DSB was obtained.

With regard to the low homologous recombination frequencies in higher eukaryotes, recombination between homologous chromosomes by NHEJ might be preferable to homologous recombination-mediated recombination. For instance, simultaneous DSB induction in both homologous chromosomes might result in the reciprocal exchange of genetic material by NHEJ. Recently, recombination between homologous chromosomes was induced by end-joining in human cells, targeting CD44 (ref. ${ }^{58}$ ). By making use of compound heterozygous mutations and DSB induction in between those mutations, subsequent reciprocal recombination was detected by simple restoration of gene function. Both nicks and DSBs guided to both homologous chromosomes resulted in reciprocal recombination, which was validated for multiple guides. After induction of DSBs, recombination frequencies were one order of magnitude higher than after induction of nicks, and reached frequencies of around $0.1 \%$. Interestingly, nicks and DSBs that were guided to only one of the homologous chromosomes failed to stimulate recombination, indicating that NHEJ is indeed preferable to homologous recombination for the targeted induction of recombination in higher eukaryotes.

\section{CRISPR-Cas-mediated chromosome engineering in mammals and yeast}

As discussed above, the site-specific induction or global enhancement of crossovers is one of the first-choice strategies when developing ways of breaking genetic linkages. However, the CRISPR-Cas system can also be used to restructure and reshuffle chromosomes through the induction of large chromosomal rearrangements. Much progress has been made in other organisms, such as yeast and mammals, using this strategy and it might provide helpful guidance for the application of similar approaches in plants.

In mammalian cells, for example, the CRISPR-Cas system has been used to recreate oncogenic chromosomal rearrangements in vitro to study the initiation of cancer ${ }^{59,60}$. Several researchers have succeeded in the reproduction of chromosomal translocations in mammalian cells using CRISPR-Cas ${ }^{60-62}$. For example, the translocations $t(8 ; 21)$ and $t(11 ; 22)$, which cause Ewing's sarcoma and acute myeloid leukaemia, respectively, were recreated successfully ${ }^{61}$. Another example of an oncogenic chromosomal rearrangement is an inversion on the human chromosome 2 , which, due to the fusion of the genes $E M L 4$ and $A L K$, leads to the formation of an

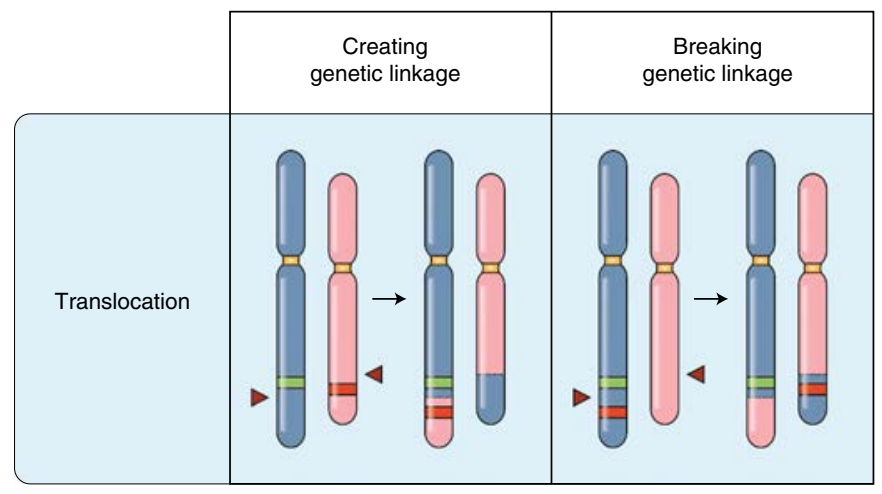

Fig. 3 | Control of genetic linkages by targeted induction of reciprocal translocations. The targeted induction of reciprocal translocations between heterologous chromosomes provides a novel type of control over genetic linkages. Traits (green and red boxes) can be either genetically linked or separated by exchanging the respective arms between different chromosomes. Red arrowheads indicate CRISPR-Cas-mediated DSBs.

abnormal fusion protein. It is associated with a certain type of lung cancer and has been induced successfully in mouse and human cells using CRISPR-Cas ${ }^{63}$. Thus, CRISPR-Cas has already been shown in several instances to be applicable for chromosome engineering in mammals.

Impressive progress has also been made on approaches to restructuring chromosomes in yeast using CRISPR-Cas. In two remarkable experiments, Shao et al. and Luo et al. were able to reduce the chromosome number of Saccharomyces cerevisiae, from 16 to 1 and 2 chromosomes, respectively ${ }^{64,65}$, by prompting a number of CRISPR-Cas-induced translocations and fusions. According to Luo et al., the 2-chromosome strain might qualify as a distinct species by classical biological species definition, because backcrossing with the 16-chromosome wild type was not possible. In addition, instances of chromosome shuffling through the CRISPR-Cas-mediated generation of chromosomal rearrangements have been reported in yeast ${ }^{66,67}$.

\section{Reversion of natural chromosomal inversions can restore genetic exchange in recombination-dead regions in plants}

Many crop plants have been found to carry chromosomal rearrangements ${ }^{29,68-70}$. The sequence diversity within a crop species can be detected by chromosome-scale sequence assemblies to capture the 'pan-genome' ${ }^{68}$. In a genome alignment of current barley elite germplasm, predominantly large inversion polymorphisms of more than $5 \mathrm{Mb}$ were detected ${ }^{68}$. Therefore, a lot of genetic material is currently inaccessible for breeding, meaning that a technique to induce or reverse evolutionary-derived chromosomal rearrangements in a targeted manner would be of great value for breeders. In 2019, Schmidt et al. were able to show that targeted inversions of up to $18 \mathrm{~kb}$ could be induced in $A$. thaliana by using Cas9 from Staphylococcus aureus under egg-cell-specific expression ${ }^{71}$. In a follow-up study, Schmidt et al. went one step further and reversed an evolutionary-derived inversion in A. thaliana, the well-known heterochromatic knob hk4S, which has a size of $1.17 \mathrm{Mb}$ and is carried by many accessions ${ }^{23,28,72}$. The authors detected 7 independent inversion events in the progeny of 38 primary transformants. This proof-of-concept study suggested that it should also be possible to induce or reverse inversions in the $\mathrm{Mb}$ range in crop plants. Another of the authors' aims was to show whether meiotic recombination could be restored in hybrids that harbour the reverted knob in a heterozygous state. Previously, it had been shown that genetic exchange between the knob-carrying accession Columbia 


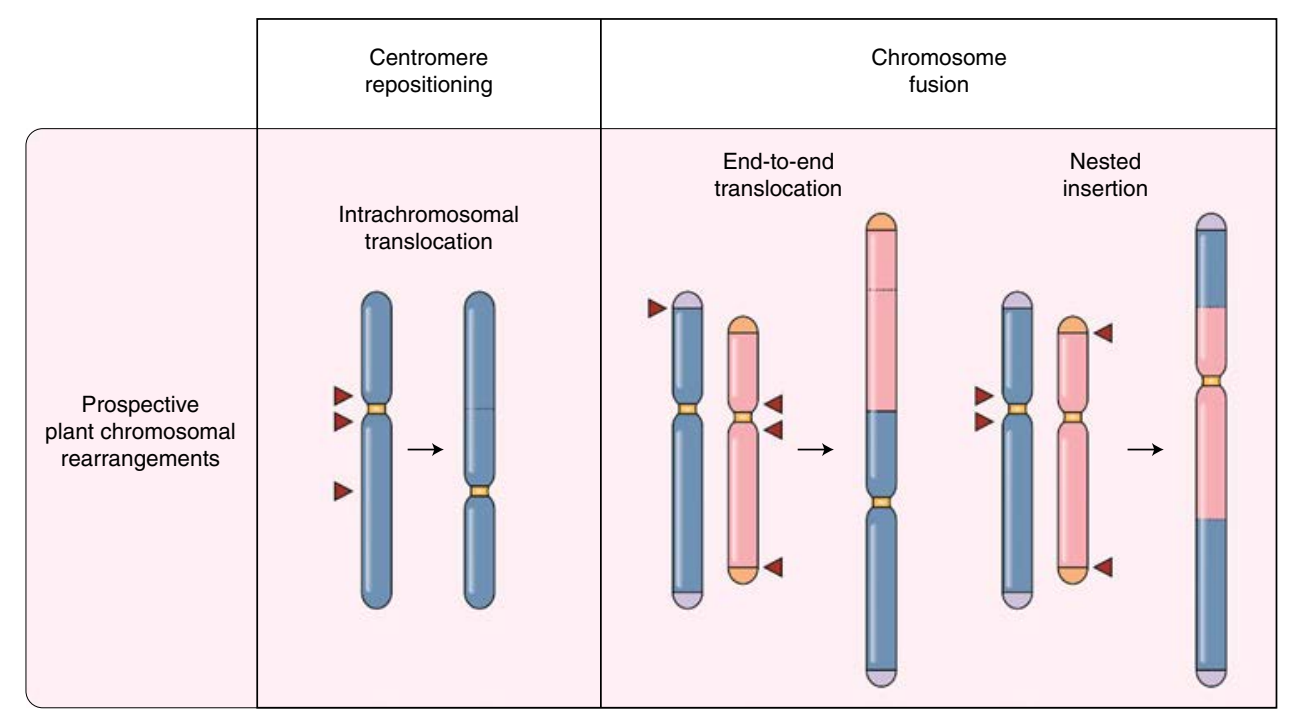

Fig. 4 | Future perspective on chromosome engineering in plants. Different chromosomal rearrangements that have occurred naturally during evolution could be taken as inspiration for what may be possible in CRISPR-Cas-mediated chromosome engineering in future. In particular, centromere repositioning and chromosome fusions are frequently occurring natural chromosomal rearrangements, and are well described. Using CRISPR-Cas, centromere repositioning could be achieved by intrachromosomal translocations, induced by three DSBs (red arrowheads). Chromosome fusions could be accomplished through end-to-end translocation or nested chromosome insertion by inducing four DSBs to remove redundant telomeres and centromeres.

and a knob-less accession, Landsberg erecta could not be detected in the rearranged $\operatorname{area}^{73}$. To test their hypothesis, Schmidt et al. conducted a SNP-based recombination assay and found that crossover rates could indeed be restored in the hybrids ${ }^{28}$. This important finding suggests that reversing evolutionary-derived chromosomal rearrangements can indeed unlock the genetic material within the chromosomal rearrangement for meiotic recombination. In keeping with this study, the first CRISPR-Cas-mediated inversion in a crop plant, specifically in an elite maize inbred line, was recently obtained $^{29}$. The 75.5-Mb pericentric inversion was located on chromosome 2, spanning nearly one-third of the chromosome ${ }^{29}$. The two $\mathrm{T}_{0}$ regenerants that harboured the inversion and transmission were isolated and segregation of the event was confirmed, as $45 \%$ of the $\mathrm{T}_{1}$ progeny plants were positive for the inversion. The findings of this study suggest that chromosome engineering will also be possible in other crop plants that are susceptible to transformation. Now that the way has been paved for chromosome engineering approaches in crops, rapid progress is likely in the near future. The induction or reversion of chromosomal inversions can be used in crop improvement to break genetic linkage groups through physical separation or to restore crossovers in the formerly rearranged areas (Fig. 2). Conversely, the induction of inversions could be used to stabilize genetic linkages between favourable traits (Fig. 2). Such artificial inversions would make the region inaccessible for recombination between the homologous chromosomes during meiosis.

\section{Induction of reciprocal translocations in plants}

As with inversions, large translocations are commonly found in crops and can lead to a reduction of meiotic recombination ${ }^{25,69}$. Reciprocal translocations in plants can cause semi-sterility and may be involved in male sterility, female sterility and sometimes both types of sterility ${ }^{74}$. Consequently, the targeted induction of translocations will probably be an important tool for breeders for breaking or stabilizing genetic linkages. Linkages can be broken through physical separation or be established by combining favourable genes from different chromosomes on the same chromosome (Fig. 3). The first targeted induction of reciprocal translocations was recently achieved in plants. Beying et al. were able to induce a reciprocal chromosomal translocation in A. thaliana between chromosome 1 and 2, and between chromosome 1 and 5. The translocated fragments had a size of around $1 \mathrm{Mb}$ and $0.5 \mathrm{Mb}$. The translocations were heritable and translocation frequencies of up to $2.5 \%$ in the wild-type background, and up to $3.75 \%$ in the classical NHEJ mutant $k u 70$, were detected in individual $\mathrm{T}_{2}$ lines. This proof-of-concept study provides grounds for optimism that the same will be possible in crop plants in the future.

\section{Potentials of restructuring the plant chromosome}

As it is now possible to change the gene order within and between chromosomes, it is tempting to speculate about what else might be achievable in the near and the more distant future. Nevertheless, one should keep in mind that despite recent progress, the technology of chromosome engineering in plants is still in its infancy. Compared with the induction of DSB-based mutations, chromosomal rearrangements are very infrequent. Indeed, a quantitative analysis of the formation of reciprocal translocations detected translocation events in 1 out of 10,000 plant cells, indicating that chromosomal rearrangements are induced in only a very small fraction of repair reactions ${ }^{27}$. In the wild type, host proteins of the classical NHEJ (cNHEJ) pathway keep the corresponding broken DNA ends in close proximity after a DSB to safeguard their re-ligation, thereby blocking the formation of chromosomal rearrangements. Therefore, it might be favourable to introduce chromosomal rearrangements into a cNHEJ-mutant background. It has been shown that, in the absence of the Ku70 protein, a central player of cNHEJ, inversion frequencies and translocation frequencies are both enhanced in Arabidopsis, the latter by up to five times ${ }^{27}$. In the absence of $\mathrm{Ku} 70$, the alternative NHEJ pathway, which has no preference for keeping the broken DNA ends together, takes over DSB repair. Thus, the use of DSB repair mutants could enhance the chances of obtaining chromosomal rearrangements. However, the downside of such an approach is that other, unwanted genomic changes might occur due to an overall repair deficiency. Additionally, the use of Cas nucleases and sgRNAs, which have been optimized for efficient DSB induction in the respective plant species, is important in enhancing chromosomal-rearrangement frequencies ${ }^{75,76}$. 
Moreover, an efficient screening protocol is required to detect rare chromosomal-rearrangement events. In Arabidopsis, a bulk screening protocol, originally developed to detect rare gene targeting events $^{77}$, has been applied successfully.

Throughout the course of evolution, several kinds of chromosomal rearrangements have arisen that could conceivably serve as models for the kind of genomic changes that may be achievable by CRISPR-Cas-mediated chromosome engineering. Chromosomal rearrangements have often occurred after polyploidization events that were followed by dysploid changes, ultimately resulting in a diploid chromosome set ${ }^{78}$. Therefore, it should be possible to achieve a reduction in chromosome numbers, either by nested chromosome insertions or by end-to-end translocation, which are both well-documented natural chromosomal rearrangements. Polyploid species such as wheat or potato might be interesting candidates for these types of chromosome engineering approaches. Moreover, centromere repositioning occurred frequently during plant genome evolution ${ }^{78}$ and was recently documented in vivo in corn after gamma irradiation ${ }^{79}$. Since it has been possible to induce inversions and translocations, which occur with high frequency on the evolutionary scale, it might also be possible to mimic nature by changing centromere potions and chromosome numbers in the not-too-distant future (Fig. 4). However, it has to be kept in mind that by inducing multiple DSBs simultaneously, other unintended genomic changes might occur, which need to be selected against. Combined with currently available approaches, this would also allow basic questions to be addressed on how different positions on a chromosome might influence chromatin state and gene expression. Moreover, through chromosome engineering, it might be possible to reconstruct chromosome sets of the ancestors of current plant species.

\section{The creation of synthetic chromosomes and new plant species}

Since the 2000s, a novel way of manipulating genomes has emerged: the construction of synthetic chromosomes. The first synthetic chromosomes were of viral origin, due to their small size ${ }^{80}$. This success was followed by the creation of synthetic bacterial chromosomes in a variety of strains, as well as the creation of synthetic yeast chromosomes ${ }^{81}$. However, the transfer of fully synthesized chromosomes from other organisms into plants has faced various technical barriers-for example, limitations in the transformation process. Both common plant transformation methods, Agrobacterium-mediated transformation and biolistic transformation, are limited in the amount of DNA they are able to transfer. Agrobacterium-mediated transfer is dependent on the standard Escherichia coli vector capacities ${ }^{82}$ of about $150 \mathrm{~kb}$. Conversely, biolistic transformation is able to transfer up to $1,100 \mathrm{~kb}$, but it can cause severe damage to the transgene and host genome and is, usually, unable to deliver intact molecules of more than a few kilobases ${ }^{83,84}$. Additionally, synthetic chromosomes must be of a certain size to support at least one crossover during meiosis. This is necessary to ensure the correct separation of the chromatids; otherwise, premature sister chromatid separation might occur, which can result in degradation and the loss of genetic material ${ }^{82}$. Since these aspects currently limit the chances of creating synthetic plant chromosomes from scratch, the next step in the generation of synthetic plant chromosomes will probably be achieved by CRISPRCas-mediated chromosome engineering, combined with the incorporation of smaller stretches of synthetic DNA.

However, the creation of new plant species will be within reach much sooner. The first success in the thorough remodelling of a eukaryotic genome was achieved in 2019, when Shao et al. and Luo et al. were able to produce reproductively isolated single-chromosome and two-chromosome $S$. cerevisiae cells via CRISPR-Cas-mediated chromosome engineering ${ }^{64,65}$. Considering the great progress that has been made in the CRISPR-Cas-based restructuring of plant chromosomes, it might be possible to adopt a similar strategy in the creation of a new plant species by means of reproductive isolation. A reduction of the chromosome number or a combination of different types of chromosomal rearrangements could lead to reproductive isolation between the engineered plant and the wild-type parent. In that case, the engineered line might qualify as a new plant species. This could be an interesting strategy for prevention of undesired outcrossing of crop plants with wild relatives. The setup of chromosome engineering in plants has unlocked exciting and unprecedented possibilities in the field of crop improvement and will help broaden the understanding of evolution and genetics.

Received: 15 February 2021; Accepted: 31 March 2021; Published online: 6 May 2021

\section{References}

1. Bailey-Serres, J., Parker, J. E., Ainsworth, E. A., Oldroyd, G. E. D. \& Schroeder, J. I. Genetic strategies for improving crop yields. Nature 575, 109-118 (2019).

2. Wolter, F., Schindele, P. \& Puchta, H. Plant breeding at the speed of light: the power of CRISPR/Cas to generate directed genetic diversity at multiple sites. BMC Plant Biol. 19, 176 (2019).

3. Lewis, R. S. \& Rose, C. Agronomic performance of tobacco mosaic virus-resistant tobacco lines and hybrids possessing the resistance gene $N$ introgressed on different chromosomes. Crop Sci. 50, 1339-1347 (2010).

4. Li, J., Chitwood, J., Menda, N., Mueller, L. \& Hutton, S. F. Linkage between the I-3 gene for resistance to Fusarium wilt race 3 and increased sensitivity to bacterial spot in tomato. Theor. Appl. Genet. 131, 145-155 (2018).

5. Zhang, Y., Pribil, M., Palmgren, M. \& Gao, C. A CRISPR way for accelerating improvement of food crops. Nat. Food 1, 200-205 (2020).

6. Zaidi, S. S.-E.-A., Mahas, A., Vanderschuren, H. \& Mahfouz, M. M. Engineering crops of the future: CRISPR approaches to develop climate-resilient and disease-resistant plants. Genome Biol. 21, 289 (2020).

7. Schindele, A., Dorn, A. \& Puchta, H. CRISPR/Cas brings plant biology and breeding into the fast lane. Curr. Opin. Biotechnol. 61, 7-14 (2020).

8. Atkins, P. A. \& Voytas, D. F. Overcoming bottlenecks in plant gene editing. Curr. Opin. Plant Biol. 54, 79-84 (2020).

9. Zhang, Y., Malzahn, A. A., Sretenovic, S. \& Qi, Y. The emerging and uncultivated potential of CRISPR technology in plant science. Nat. Plants $\mathbf{5}$, 778-794 (2019).

10. Jinek, M. et al. A programmable dual-RNA-guided DNA endonuclease in adaptive bacterial immunity. Science 337, 816-821 (2012).

11. Puchta, $\mathrm{H}$. The repair of double-strand breaks in plants: mechanisms and consequences for genome evolution. J. Exp. Bot. 56, 1-14 (2004).

12. Schmidt, C., Schindele, P. \& Puchta, H. From gene editing to genome engineering: restructuring plant chromosomes via CRISPR/Cas. aBIOTECH 1, 21-31 (2020).

13. Rönspies, M., Schindele, P. \& Puchta, H. CRISPR/Cas-mediated chromosome engineering: opening up a new avenue for plant breeding. J. Exp. Bot. 72, 177-183 (2021).

14. Thompson, S. L. \& Compton, D. A. Chromosomes and cancer cells. Chromosome Res. 19, 433-444 (2011).

15. Park, C.-Y., Sung, J. J. \& Kim, D.-W. Genome editing of structural variations: modeling and gene correction. Trends Biotechnol. 34, 548-561 (2016).

16. Brunet, E. \& Jasin, M. Induction of chromosomal translocations with CRISPR-Cas9 and other nucleases: understanding the repair mechanisms that give rise to translocations. Adv. Exp. Med. Biol. 1044, 15-25 (2018).

17. Hasty, P. \& Montagna, C. Chromosomal rearrangements in cancer: detection and potential causal mechanisms. Mol. Cell. Oncol. 1, e29904 (2014).

18. Lakich, D., Kazazian, H. H. Jr, Antonarakis, S. E. \& Gitschier, J. Inversions disrupting the factor VIII gene are a common cause of severe haemophilia A. Nat. Genet. 5, 236-241 (1993).

19. Bondeson, M. L. et al. Inversion of the IDS gene resulting from recombination with IDS-related sequences is a common cause of the Hunter syndrome. Hum. Mol. Genet. 4, 615-621 (1995).

20. Small, K., Iber, J. \& Warren, S. T. Emerin deletion reveals a common $\mathrm{X}$-chromosome inversion mediated by inverted repeats. Nat. Genet. 16, 96-99 (1997).

21. Park, C.-Y., Lee, D. R., Sung, J. J. \& Kim, D.-W. Genome-editing technologies for gene correction of hemophilia. Hum. Genet. 135, 977-981 (2016).

22. Kirkpatrick, M. \& Barton, N. Chromosome inversions, local adaptation and speciation. Genetics 173, 419-434 (2006).

23. Fransz, P. et al. Molecular, genetic and evolutionary analysis of a paracentric inversion in Arabidopsis thaliana. Plant J. 88, 159-178 (2016). 
24. Harewood, L. \& Fraser, P. The impact of chromosomal rearrangements on regulation of gene expression. Hum. Mol. Genet. 23, R76-R82 (2014).

25. Martin, G. et al. Chromosome reciprocal translocations have accompanied subspecies evolution in bananas. Plant J. 104, 1698-1711 (2020).

26. Wellenreuther, M. \& Bernatchez, L. Eco-evolutionary genomics of chromosomal inversions. Trends Ecol. Evol. 33, 427-440 (2018).

27. Beying, N., Schmidt, C., Pacher, M., Houben, A. \& Puchta, H. CRISPRCas9-mediated induction of heritable chromosomal translocations in Arabidopsis. Nat. Plants 6, 638-645 (2020).

28. Schmidt, C. et al. Changing local recombination patterns in Arabidopsis by CRISPR/Cas mediated chromosome engineering. Nat. Commun. 11, 4418 (2020).

29. Schwartz, C. et al. CRISPR-Cas9-mediated 75.5-Mb inversion in maize. Nat. Plants 6, 1427-1431 (2020).

30. Taagen, E., Bogdanove, A. J. \& Sorrells, M. E. Counting on crossovers: controlled recombination for plant breeding. Trends Plant Sci. 25, 455-465 (2020)

31. Wang, Y. \& Copenhaver, G. P. Meiotic recombination: mixing it up in plants. Annu. Rev. Plant Biol. 69, 577-609 (2018).

32. Lambing, C., Franklin, F. C. H. \& Wang, C.-J. R. Understanding and manipulating meiotic recombination in plants. Plant Physiol. 173, 1530-1542 (2017).

33. Bergerat, A. et al. An atypical topoisomerase II from archaea with implications for meiotic recombination. Nature 386, 414-417 (1997).

34. Crismani, W. et al. FANCM limits meiotic crossovers. Science 336, 1588-1590 (2012)

35. Hartung, F., Suer, S. \& Puchta, H. Two closely related RecQ helicases have antagonistic roles in homologous recombination and DNA repair in Arabidopsis thaliana. Proc. Natl Acad. Sci. USA 104, 18836-18841 (2007).

36. Séguéla-Arnaud, M. et al. Multiple mechanisms limit meiotic crossovers: TOP $3 \alpha$ and two BLM homologs antagonize crossovers in parallel to FANCM. Proc. Natl Acad. Sci. USA 112, 4713-4718 (2015).

37. Dorn, A. et al. The topoisomerase $3 \alpha$ zinc-finger domain T1 of Arabidopsis thaliana is required for targeting the enzyme activity to Holliday junction-like DNA repair intermediates. PLoS Genet. 14, e1007674 (2018).

38. Séguéla-Arnaud, M. et al. RMI1 and TOP $3 \alpha$ limit meiotic CO formation through their C-terminal domains. Nucleic Acids Res. 45, 1860-1871 (2017).

39. de Maagd, R. A. CRISPR/Cas inactivation of RECQ4 increases homeologous crossovers in an interspecific tomato hybrid. Plant Biotechnol. J. 18, 805-813 (2020).

40. Girard, C. et al. AAA-ATPase FIDGETIN-LIKE 1 and helicase FANCM antagonize meiotic crossovers by distinct mechanisms. PLoS Genet. 11, e1005369 (2015)

41. Fernandes, J. B. et al. FIGL1 and its novel partner FLIP form a conserved complex that regulates homologous recombination. PLoS Genet. 14, e1007317 (2018).

42. Zhang, P. et al. The rice AAA-ATPase OsFIGNL1 is essential for male meiosis. Front. Plant Sci. 8, 1639 (2017).

43. Mieulet, D. et al. Unleashing meiotic crossovers in crops. Nat. Plants 4 1010-1016 (2018)

44. Pyatnitskaya, A., Borde, V. \& de Muyt, A. Crossing and zipping: molecular duties of the ZMM proteins in meiosis. Chromosoma 128, 181-198 (2019).

45. Ziolkowski, P. A. et al. Natural variation and dosage of the HEI10 meiotic E3 ligase control Arabidopsis crossover recombination. Genes Dev. 31, 306-317 (2017)

46. Serra, H. et al. Massive crossover elevation via combination of HEI10 and recq4a recq4b during Arabidopsis meiosis. Proc. Natl Acad. Sci. USA 115, 2437-2442 (2018).

47. Wang, K., Wang, C., Liu, Q., Liu, W. \& Fu, Y. Increasing the genetic recombination frequency by partial loss of function of the synaptonemal complex in rice. Mol. Plant 8, 1295-1298 (2015).

48. Fernandes, J. B., Séguéla-Arnaud, M., Larchevêque, C., Lloyd, A. H. \& Mercier, R. Unleashing meiotic crossovers in hybrid plants. Proc. Natl Acad. Ssci. USA 115, 2431-2436 (2018).

49. Hartung, F., Suer, S., Knoll, A., Wurz-Wildersinn, R. \& Puchta, H. Topoisomerase $3 \alpha$ and RMI1 suppress somatic crossovers and are essential for resolution of meiotic recombination intermediates in Arabidopsis thaliana. PLoS Genet. 4, e1000285 (2008).

50. Mannuss, A. et al. RAD5A, RECQ4A, and MUS81 have specific functions in homologous recombination and define different pathways of DNA repair in Arabidopsis thaliana. Plant Cell 22, 3318-3330 (2010).

51. Schröpfer, S., Kobbe, D., Hartung, F., Knoll, A. \& Puchta, H. Defining the roles of the N-terminal region and the helicase activity of RECQ4A in DNA repair and homologous recombination in Arabidopsis. Nucleic Acids Res. 42, 1684-1697 (2014).

52. Knoll, A. et al. The Fanconi anemia ortholog FANCM ensures ordered homologous recombination in both somatic and meiotic cells in Arabidopsis. Plant Cell 24, 1448-1464 (2012).
53. Peciña, A. et al. Targeted stimulation of meiotic recombination. Cell 111, 173-184 (2002)

54. Sarno, R. et al. Programming sites of meiotic crossovers using Spol1 fusion proteins. Nucleic Acids Res. 45, e164 (2017).

55. Yelina, N. E., Gonzalez-Jorge, S., Hirsz, D., Yang, Z. \& Henderson, I. R. CRISPR targeting of MEIOTIC-TOPOISOMERASE VIB-dCas9 to a recombination hotspot is insufficient to increase crossover frequency in Arabidopsis. Preprint at bioRxiv https://doi.org/10.1101/2021.02.01.429210 (2021)

56. Filler Hayut, S., Melamed Bessudo, C. \& Levy, A. A. Targeted recombination between homologous chromosomes for precise breeding in tomato. Nat. Commun. 8, 15605 (2017).

57. Ben Shlush, I. et al. CRISPR/Cas9 induced somatic recombination at the CRTISO locus in tomato. Genes 12, 59 (2020).

58. Davis, L., Khoo, K. J., Zhang, Y. \& Maizels, N. POLQ suppresses interhomolog recombination and loss of heterozygosity at targeted DNA breaks. Proc. Natl Acad. Sci. USA 117, 22900-22909 (2020).

59. Cheong, T.-C., Blasco, R. B. \& Chiarle, R. The CRISPR/Cas9 System as a tool to engineer chromosomal translocation in vivo. Adv. Exp. Med. Biol. 1044, 39-48 (2018).

60. Torres-Ruiz, R. et al. Efficient recreation of $\mathrm{t}(11 ; 22)$ EWSR1-FLI1 ${ }^{+}$in human stem cells Using CRISPR/Cas9. Stem Cell Rep. 8, 1408-1420 (2017).

61. Torres, R. et al. Engineering human tumour-associated chromosomal translocations with the RNA-guided CRISPR-Cas9 system. Nat. Commun. 5, 3964 (2014).

62. Lagutina, I. V. et al. Modeling of the human alveolar rhabdomyosarcoma Pax3-Foxo1 chromosome translocation in mouse myoblasts using CRISPRCas9 nuclease. PLoS Genet. 11, e1004951 (2015).

63. Choi, P. S. \& Meyerson, M. Targeted genomic rearrangements using CRISPR/ Cas technology. Nat. Commun. 5, 3728 (2014).

64. Luo, J., Sun, X., Cormack, B. P. \& Boeke, J. D. Karyotype engineering by chromosome fusion leads to reproductive isolation in yeast. Nature $\mathbf{5 6 0}$, 392-396 (2018)

65. Shao, Y. et al. Creating a functional single-chromosome yeast. Nature 560, 331-335 (2018)

66. Fleiss, A. et al. Reshuffling yeast chromosomes with CRISPR/Cas9. PLoS Genet. 15, e1008332-e1008332 (2019).

67. Yadav, V., Sun, S., Coelho, M. A. \& Heitman, J. Centromere scission drives chromosome shuffling and reproductive isolation. Proc. Natl Acad. Sci. USA 117, 7917-7928 (2020).

68. Jayakodi, M. et al. The barley pan-genome reveals the hidden legacy of mutation breeding. Nature 588, 284-289 (2020).

69. Walkowiak, S. et al. Multiple wheat genomes reveal global variation in modern breeding. Nature 588, 277-283 (2020).

70. Crow, T. et al. Gene regulatory effects of a large chromosomal inversion in highland maize. PLoS Genet. 16, e1009213 (2020).

71. Schmidt, C., Pacher, M. \& Puchta, H. Efficient induction of heritable inversions in plant genomes using the CRISPR/Cas system. Plant J. 98 577-589 (2019).

72. Fransz, P. F. et al. Integrated cytogenetic map of chromosome arm $4 \mathrm{~S}$ of $A$. thaliana: structural organization of heterochromatic knob and centromere region. Cell 100, 367-376 (2000).

73. Drouaud, J. et al. Variation in crossing-over rates across chromosome 4 of Arabidopsis thaliana reveals the presence of meiotic recombination "hot spots". Genome Res. 16, 106-114 (2006).

74. Zhang, Y., Cheng, Z., Ma, J., Xian, F. \& Zhang, X. Characteristics of a novel male-female sterile watermelon (Citrullus lanatus) mutant. Sci. Horticulturae 140, 107-114 (2012)

75. Steinert, J., Schiml, S., Fauser, F. \& Puchta, H. Highly efficient heritable plant genome engineering using Cas9 orthologues from Streptococcus thermophilus and Staphylococcus aureus. Plant J. 84, 1295-1305 (2015).

76. Schindele, P. \& Puchta, H. Engineering CRISPR/LbCas12a for highly efficient, temperature-tolerant plant gene editing. Plant Biotechnol. J. 18, 1118-1120, https://doi.org/10.1111/pbi.13275 (2020).

77. Wolter, F., Klemm, J. \& Puchta, H. Efficient in planta gene targeting in Arabidopsis using egg cell-specific expression of the Cas9 nuclease of Staphylococcus aureus. Plant J. 94, 735-746 (2018).

78. Mandáková, T. \& Lysak, M. A. Post-polyploid diploidization and diversification through dysploid changes. Curr. Opin. Plant Biol. 42, 55-65 (2018).

79. Liu, Y. et al. Rapid birth or death of centromeres on fragmented chromosomes in maize. Plant Cell 32, 3113-3123 (2020).

80. Wimmer, E., Mueller, S., Tumpey, T. M. \& Taubenberger, J. K. Synthetic viruses: a new opportunity to understand and prevent viral disease. Nat. Biotechnol. 27, 1163-1172 (2009).

81. Coradini, A. L. V., Hull, C. B. \& Ehrenreich, I. M. Building genomes to understand biology. Nat. Commun. 11, 6177 (2020).

82. Dawe, R. K. Charting the path to fully synthetic plant chromosomes. Exp. Cell. Res. 390, 111951 (2020). 
83. Liu, J. et al. Genome-scale sequence disruption following biolistic transformation in rice and maize. Plant Cell 31, 368-383 (2019).

84. Zhang, H. et al. Stable integration of an engineered megabase repeat array into the maize genome. Plant J. 70, 357-365 (2012).

\section{Acknowledgements}

We thank D. Donahey for proofreading the manuscript. This work was supported by the European Research Council (Advanced grant: ERC-2016-AdG_741306 CRISBREED).

\section{Author contributions}

All authors wrote the manuscript. A.D. and P.S. designed the figures. P.S. created the figures.

\section{Competing interests}

The authors declare no competing interests. 


\section{Repository KITopen}

Dies ist ein Postprint/begutachtetes Manuskript.

Empfohlene Zitierung:

Rönspies, M.; Dorn, A.; Schindele, P.; Puchta, H.

CRISPR-Cas-mediated chromosome engineering for crop improvement and synthetic biology. 2021. Nature Plants, 7. doi: $10.5445 /$ IR/1000133197

Zitierung der Originalveröffentlichung:

Rönspies, M.; Dorn, A.; Schindele, P.; Puchta, H.

CRISPR-Cas-mediated chromosome engineering for crop improvement and synthetic biology.

2021. Nature Plants, 7, 566-573.

doi:10.1038/s41477-021-00910-4 